\title{
Surface-enhanced Raman scattering microfluidic sensor
}

Cite this: RSC Advances, 2013, 3, 13015

Received 4th February 2013,

Accepted 12th April 2013

DOI: $10.1039 /$ c3ra40610e

www.rsc.org/advances

\author{
Qing-ling Li, ${ }^{* a}$ Bo-wei $\mathrm{Li}^{\mathrm{b}}$ and Yun-qing Wang ${ }^{\mathrm{b}}$
}

\begin{abstract}
Surface-enhanced Raman scattering (SERS) is a highly sensitive detection technique used to provide information about chemical and biological trace analytes. The confocal Raman microscope makes it possible to monitor small samples in a narrow microfluidic channel, despite the fact that the scattering intensity of the Raman signal is very weak. Recently, microfluidic chip devices coupled with on-chip SERS detection method and their applications to sensitive chemical and biological analyses have attracted significant attention. In this mini-review, highlights of advances in SERS techniques, microfluidic devices and their applications on the biological/environmental analysis of minute analytes within the recent years are provided to illustrate the impact of this rapidly growing area of research.
\end{abstract}

\section{Introduction}

Raman spectroscopy is a well-known analytical method that offers great advantages for probing the structural properties of a compound on a microscopic scale. However, Raman scattering is an extremely inefficient process with low scattering cross-sections that are approximately 14 orders of magnitude smaller than the absorption cross-sections of fluorescent dye molecules. Surface-enhanced Raman scatter-

${ }^{a}$ The Key Lab in Molecular and Nano-materials Probes of the Ministry of Education of China, College of Chemistry, Chemical Engineering and Materials Science, Shandong Normal University, Jinan 250014, China. E-mail: liql@sdnu.edu.cn; Fax: 86-531 86182544; Tel: 86-531 86182544

${ }^{b}$ Key Laboratory of Coastal Zone Environmental Processes and Ecological Remediation, Yantai Institute of Coastal Zone Research, Chinese Academy of Sciences, Yantai 264003, China

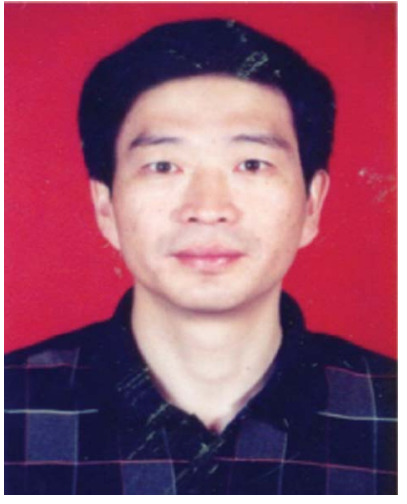

Qing-ling Li
Qing-ling Li received his B.S. degree in electrical engineering from Shandong Industrial University, Jinan, China, in 1984. From 1984 to 1999 he was an engineer, and from 1999 to 2005 he was a Senior Engineer in the Shandong Chemical Engineering Institute, Jinan, China. Science 2005, He has been a Senior Engineer in the Department of Chemistry, Chemical Engineering and Materials Science, College of Shandong Normal University, Jinan, China. His research interests include analytical instrumentation (electrophoresis, microfluidic analysis systems detection). ing (SERS) spectroscopy has shown promise in overcoming the low sensitivity problems inherent in conventional Raman spectroscopy. ${ }^{1}$ Using the SERS technique, it is known that the detection sensitivity of nanoparticle ensemble averages is enhanced up to 6-10 orders of magnitude over conventional Raman spectroscopy. ${ }^{2}$ In addition, it is also reported that it is possible to obtain an enormous Raman enhancement using hot spots on aggregated silver particle clusters. ${ }^{3}$ The observed enhancement factors are typically estimated to be 8-10 orders ${ }^{4}$ much larger than the ensemble-averaged values. As a result, the SERS technique provides a sensitivity that is comparable with fluorescence detection. Furthermore, it is possible to simultaneously detect multiple analytes using SERS because the signals are much narrower than fluorescence bands. The confocal Raman microscope makes it possible to monitor small samples in a narrow microfluidic channel,

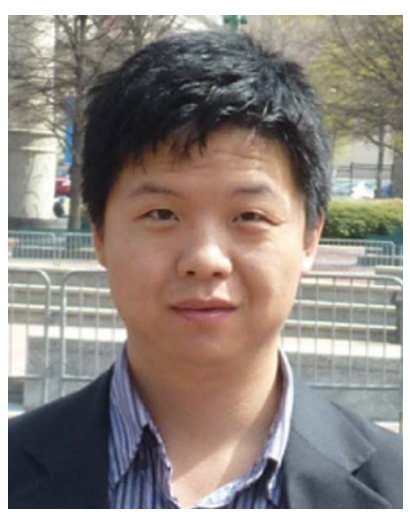

Bo-wei Li
Bo-wei Li received his B.S. degree in chemistry from the Department of Chemistry of Xiamen University, Xiamen, China, in 2001 and his Ph.D degree from Dalian Institute of Chemical Physics, Chinese Academy of Science (CAS), Dalian, China in 2009. From 2009 to 2011, he worked as a postdoctoral fellow in the Department of Chemistry and Biochemistry, Florida State University, FL, USA. In August, 2011 he joined the Laboratory of Environmental Microanalysis and Monitoring in Yantai Institute of Coastal Zone Research, CAS, as an assistant professor. His current research interests focus on microfluidic devices, optical microscopic/SERS detection and paper microfluidics. 
despite the fact that the scattering intensity of Raman signals is very weak. SERS detection in a microfluidic channel has some advantages over conventional SERS detection under static conditions. This is because the measurement under flowing conditions prevents problems of variable mixing times, scattering geometry, localized heating, and photo dissociation. ${ }^{5}$ For on-chip SERS detection in a fluidic channel, it is known that flow conditions yield more reproducible results than static conditions because of the more consistent geometries and heat dissipation properties. ${ }^{6}$ Many research papers on SERS detection for various biological and environmental analytes in a microfluidic channel have been published. ${ }^{7-9}$ This mini-review mainly deals with recent advances in SERS detection for microfluidic chips. Highlights of advances in SERS techniques, microfluidic devices and their applications on the biological/environmental analysis of minute analytes within the recent years are provided to illustrate the impact of this rapidly growing area of research.

\section{SERS-based microfluidic systems}

The SERS-based microfluidic systems, i.e. SERS detection in combination with microfluidic technology, optimization of the SERS experimental conditions as well as instrumental setup are very important. Under ordinary sampling conditions, the quantitative application of SERS is known to be difficult because it is hard to control the experimental conditions, such as the degree of aggregation, the different sizes of metal colloids, and the inhomogeneous distributions of molecules on the metal surface. ${ }^{5,6,10}$ However, a highly precise quantitative measurement can be obtained if the continuous flow and homogeneous mixing conditions between analytes and metal nanoparticles are maintained. For this purpose, functional microfluidic channels with micromixers are necessary for the efficient mixing of analytes and aggregated metal colloids. Under the optimal conditions of using an efficiently designed mixing channel and a stable flow velocity, quantita-

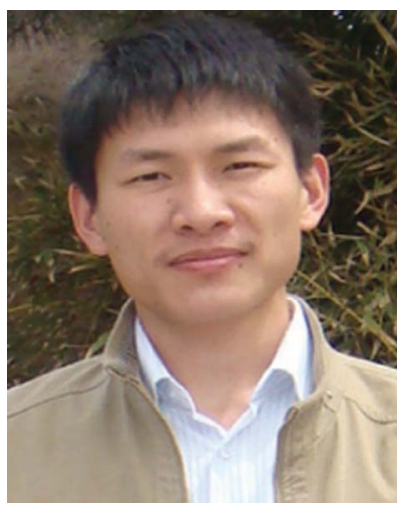

Yun-qing Wang got his Ph.D. in Pharmaceutical Analysis from China Pharmaceutical University, Nanjing, China, in 2009. In the same year, he joined the Laboratory of Environmental Microanalysis and Monitoring of Dr. Lingxin Chen in Yantai Institute of Coastal Zone Research, Chinese Academy of Sciences, as a research assistant. His current research interests focus on the development of SERS and noble metal nanoparticle-based optical sensors and the applications in bioimaging. tive SERS detection of analytes can be achieved under flow conditions. This analytical method provides fast and reproducible results for the quantitative measurement of analytes at low detection levels. The instrumental setup of the Raman microscope is another important factor. Among the various types of Raman instruments, the confocal Raman microscope has many advantages for microfluidic applications. The microscope makes it possible to investigate small samples in a narrow microfluidic channel, despite the fact that the scattering intensity of Raman signals is very weak. Here the advances in microfluidic micromixers, a synthesis of stable metal nanocolloids and the instrumental setup of the Raman microscope will be summarized in detail.

\subsection{Micromixer}

Unlike the well-controlled nanostructures formed by electronbeam lithography or nanosphere lithography which allow for good reproducibility, ${ }^{11-14}$ in the case of a colloidal solution, it is difficult for quantitative application of SERS because it is hard to control the experimental conditions, such as the degree of aggregation, the different sizes of metal colloids, and especially the inhomogeneous distributions of molecules on the metal surface. Therefore, it is very important to obtain an efficient mixing between metal nanocolloids and analytes for reproducible SERS detection. Microfluidics is the science and technology of manipulating and analyzing fluid flow in structures of sub-millimetre dimensions. ${ }^{15-17}$ This field is particularly relevant for the development of lab-on-chip microfluidic devices, ${ }^{18,19}$ which can be pictured as credit card-sized fluidic systems containing microchannels and chambers in which processes such as mixing and routing of the liquids, separation, reaction and detection of individual components present in these liquids are integrated. Many different physical phenomena such as light, electricity, magnetism and heat can be used to manipulate fluids on a sub-millimetre scale. ${ }^{20,21}$

The combination of the SERS technique with lab-on-a-chip provides potential reproducible results for the quantitative measurement of analytes. The characteristic dimensions of the internal structures of microfluidic devices typically range from the sub-micrometer to the sub-millimeter level. The Reynolds number $(R e)$ is generally low and the flows are non-turbulent due to the small channel sizes ${ }^{22}$ while the channel size is often too large for molecular diffusion to effectively mix within a reasonable time, which is reflected by the Péclet number (Pé) being large. ${ }^{23}$ A special challenge in microfluidic systems is to create efficient mixing flows, since it is almost impossible to generate turbulence. Thus, rapid mixing of fluids at the micrometer scale is a challenging problem because it is difficult to generate a turbulent flow in a microfluidic channel in the low $R e$ regime. To obtain rapid and highly efficient mixing of the fluids flowing through a microfluidic channel, special strategies for micromixers must therefore be followed, which is important for various applications of microfluidic devices. An approach is to create repeatedly stretching and folding flow patterns, leading to so-called chaotic advection that will result in effective mixing. Quantitative analysis of analytes based on intensity changes of the SERS signal is 

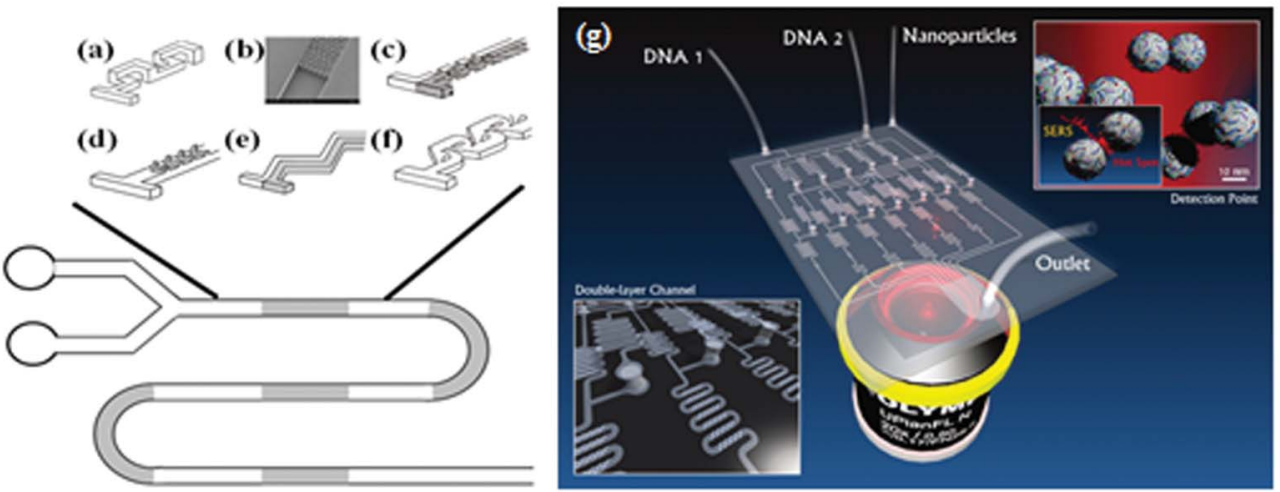

Fig. 1 Schematic illustration of a microfluidic channel for the SERS detection with different mixing manner with chaotic advection at high Reynolds numbers. (a) C-shape channel; $;{ }^{26}$ (b) pillar array channel; ${ }^{27}$ (c) obstacles on the channel's wall;; ${ }^{29}$ (d) staggered-herringbone grooves; ${ }^{28}$ (e) zig zag-shaped channel; ${ }^{30}$ ( $f$ ) modified Tesla structure; ${ }^{26}$ (Fig. (a)-(f) copyright 2008 John Wiley \& Sons, Inc.); (g) a SERS-based micro-network gradient chip for detection of DNA oligonucleotides. ${ }^{36}$ Copyright 2012 Royal Society of Chemistry.

possible by virtue of an efficient mixing channel design and an optimal flow velocity. Nguyen and $\mathrm{Wu}^{24}$ presented a review and classification of the relatively large number of micromixers proposed previously. The existing micromixers can be divided into two general classes, namely passive and active micromixers. Here, the advances of micromixers are presented although some of them are just concept-based works, and there is a long way to go for them to be used for SERS-based microsystems.

Passive micromixers do not require an external pump and energy, and the mixing process relies entirely on chaotic advection or diffusion. In contrast to active micromixers, passive micromixers are most widely used in laminar flow mixing because of their simplicity, operational applicability and have the potential to be integrated into a total microfluidic system. To obtain an efficient mixing, a miniaturized microfluidic device with special geometrical features should be designed for the generation of repeated mixing flow patterns, which will result in chaotic advection and therefore effective mixing. Many different types of passive micromixers, such as branching channel, ${ }^{25}$ three-dimensional C-shape channel, ${ }^{26}$ pillar array channel, ${ }^{27}$ and serpentine-shaped channel such as groove-shaped, ${ }^{28}$ obstacles on the channel's wall, ${ }^{29}$ modified Tesla ${ }^{26}$ and zigzag-shaped channels ${ }^{30}$ have been reported for the fast and efficient mixing of two confluent streams. Recently, a high density pillar obstruction poly(dimethylsiloxane) (PDMS) microfluidic mixer was fabricated and estimated the mixing behavior of confluent streams of three different sets of miscible solutions using a fluorescence microscope. ${ }^{31}$ This passive mixer improves the mixing efficiency by increasing the contact area between two confluent laminar flows by using multiple splitting and reunification. Compared with previous similar reports on pillar obstruction-type passive mixers, ${ }^{32,33}$ the high density pillar channels can obtain a high throughput and a fast mixing efficiency at very low $R e$, which will benefit a wide range of labon-a-chip applications. Recently, Kwang proposed a programmable mixing gradient chip based-on chip's different design. ${ }^{34}$ In addition, Choo's group relied on this theory and realized the SERS-based immunoassay and DNA detection. ${ }^{35,36}$ As shown in Fig. 1, this micromixer could be applied as an efficient microfluidic channel for SERS detection of the chemical and biological analytes.

Here we want to emphasize an important passive micromixing technique proposed by Song et al. ${ }^{37}$ who used a twophase liquid system consisting of aqueous droplets in an oily carrier liquid. By transporting the droplets through winding microchannels, an internal flow field is generated inside the droplets and the fast mixing occurs within the droplets. Now, the microdroplet channels are well-known as droplet-based microfluidic systems, which have gained increasing popularity in recent years. Such systems allow the generation and manipulation of monodisperse, nanoliter-sized liquid droplets within an immiscible carrier fluid in a high-throughput manner. ${ }^{38-43}$ Compared to single-phase flow, the localization of reagents within discrete and encapsulated droplets enhances mixing, minimizes residence time distributions and affords ultra-high analytical throughput. ${ }^{37}$ Importantly, the formed droplets might be fused, ${ }^{44}$ sub-divided, sorted, ${ }^{45}$ isolated or incubated, ${ }^{46}$ allowing complex chemical/biological processing in an automated fashion. In SERS-based microfluidic systems, it is significant to note that the deposition of metal nanoparticle aggregates on channel surfaces (the memory effect) is ameliorated in the high surface-to-volume ratio environments typical of microfluidic systems, thus affecting both sensitivity and reproducibility. ${ }^{4,48}$ The droplet-based microfluidic systems can overcome part of the problem. ${ }^{49,50}$ To address this issue in part, Popp et al. ${ }^{51,52}$ have employed a two phase liquid/liquid segmented flow system in SERS measurements. The droplet-based microfluidic systems have been developed for the trace mercury (II) ions analysis in water. $^{48}$ As illustrated in Fig. 2(a) and (b), aqueous droplets containing mercury (II) ions and Rhodamine B-adsorbed gold nanoparticles (AuNPs) act as individual reaction vessels, and the aqueous droplets are encapsulated within nanoliter-sized droplets and are completely isolated by a continuous oil phase. In this way, memory effects, caused by the precipitation of nanoparticle aggregates on channel walls, are removed. ${ }^{48}$ 


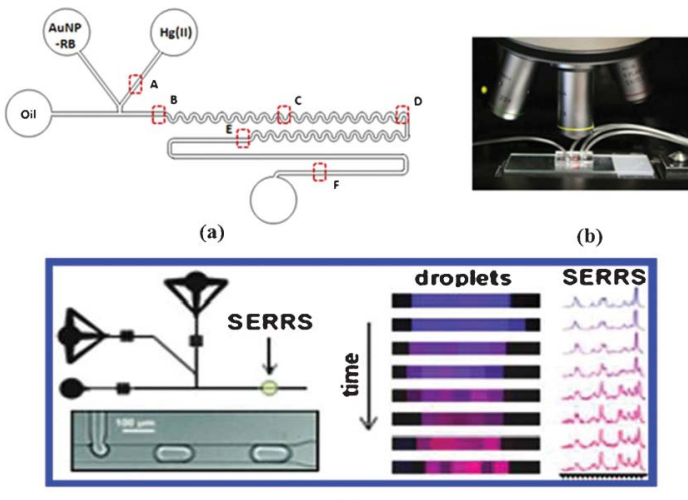

(c)

Fig. 2 (a) Schematic representation of the channel pattern used to create droplets. ${ }^{48}$ The dashed rectangles denote positions at which SERS measurements were made; (b) optical arrangement for focusing the excitation beam into the microchannel network; (c) quantitative real time characterization of individual microdroplets by SERS. ${ }^{53}$ Fig. (a), (b) and (c) were adapted with permission from ref. 48 and 53, copyright 2009 Springer-Verlag, and copyright ACS respectively).

More recently, Cecchini proposed an ultrafast and highthroughput droplet-based SERRS method that can make an efficient analysis at the submillisecond scale. ${ }^{47}$ As shown in Fig. 2(c), Christopher used a new combination of SERRS multiplexing to analyze multiple concentrations in droplets in a real-time way. ${ }^{53}$

Active micromixers that use the disturbance generated by external field forces for the mixing process, such as electrical fields, ${ }^{54,55}$ magnetic fields, ${ }^{56}$ acoustic waves $^{57}$ and even electrostatic fields, ${ }^{58}$ have been used to overcome the mixing problem in microfluidic channels. Active mixers show an excellent mixing performance and the flow control can be switched on or off, but it is relatively difficult to integrate it into a total microfluidic system because of the requirement of an external power source and controller. However, there is much progress in these kinds of micromixers. Electrical effects can be used for micromixing. El Moctar et al. ${ }^{54}$ describe a microfluidic mixer based on the electrohydrodynamic force present when the fluids to be mixed have different electrical properties and are subjected to an electrical field. Integrated electrodes are used to create an electric field perpendicular to the interface between the fluids in the microchannel, resulting in a transversal secondary flow that causes mixing. Sasaki et $a l .{ }^{55}$ used meandering electrodes in the microchannel floor to induce a transversal alternating current (AC) electroosmotic flow by which rapid mixing was achieved. Lu et al. ${ }^{56}$ reported an active mixer concept by integrating micromachined magnetic-bar mixers in microfluidic channels and chambers. These are rapidly rotated within the fluid by applying a rotating external magnetic field, which results in reasonably effective mixing. Srithanan et al. ${ }^{57}$ described a micromixing concept based on acoustic streaming, in which surface acoustic waves generated by a piezoelectric transducer induced transversal mixing flow patterns. Glasgow and Audry ${ }^{59}$ proposed an active mixer concept by applying sinusoidal pressure pulses to the microchannel through the channel inlets for active mixing. This causes an oscillating flow velocity which results in chaotic advection patterns. Very recently, den Toonder et al. ${ }^{58}$ proposed a quite versatile active microfluidic mixing concept inspired by nature, namely by microorganisms that swim through a liquid by oscillating microscopic hairs, cilia, that cover their surface. The fabricated artificial cilia consisted of electrostatically actuated polymer structures and were integrated in a microfluidic channel. Flow visualization experiments show that the integrated artificial cilia can generate substantial fluid velocities, up to $0.6 \mathrm{~mm} \mathrm{~s}^{-1}$, in open-faced microchannels. In addition, very efficient mixing is obtained using specially designed geometrical cilia configurations in a microchannel. The advantageous micromixing concept using artificial cilia has been proven to work over a wide range of Reynolds numbers, and at very high Péclet numbers. The mixing length is shorter than that of known passive mixing configurations such as the herringbone mixer. ${ }^{28}$ The mixing efficiency, expressed in mixing time per volume of mixed liquid, is better than that of existing active micromixers. With the use of patterned electrodes, the effect can be switched on or off on demand, at locations of choice. These properties make artificial cilia attractive and promising for future integrated microfluidic devices in which active fluidic control is required, particularly in lab-on-a-chip devices for bio-chemical analysis in which reagents are added and must be mixed quickly in microreaction chambers. However, the fabrication of the artificial cilia is not simple, and this adds to the total cost of producing a microfluidic device. Cheaper solutions like passive mixing may be preferred for applications that do not require active control.

Due to the high mixing efficiency of the multiphase fluids (analyte fluid, nanoparticle colloid and sometimes oil), various efficient functional microchannels have been succeeded in applications for SERS-based microsystems laminar flow mixing such as alligator teeth-shaped microfluidic channels, ${ }^{60,61}$ zig zag-shaped microfluidic channels, ${ }^{62,63}$ pillar array microfluidic channels, ${ }^{27,31}$ and microdroplet microfluidic channels. $^{48}$ In the previously mentioned functional microchannels, the nanocolloids and analytes are introduced into a channel where they are mixed with each other with high efficiency. The on-chip Raman detection in a microfluidic channel yields more reproducible results than that of a static condition. Then, the Raman signal is accumulated from the flowing stream when the mixed samples pass through a laser beam. At the laser detection point, SERS signals from different aggregates are accumulated and averaged.

To make the mixing much more effective, efficient, rapid and simple, Wang et al. ${ }^{64}$ developed a simple optofluidic molecule/particle trapping device that can improve the SERS signal. This device has a pinched and step microfluidicnanochannel junction where metallic nanoparticles and target molecules in aqueous solution can be reproducibly trapped to form nanoparticle-molecule SERS clusters using the capillary force in the channel. This optofluidic approach has three advantages. Firstly, it has higher sensitivity because of an increased local density of nanoparticle/target molecules. Secondly, the device does not require chemical agents or salts to initiate the aggregation of nanoparticles. Thirdly, the device has higher reproducibility because the SERS active clusters are 
Table 1 The different shapes of gold/silver nanoparticles and their applications

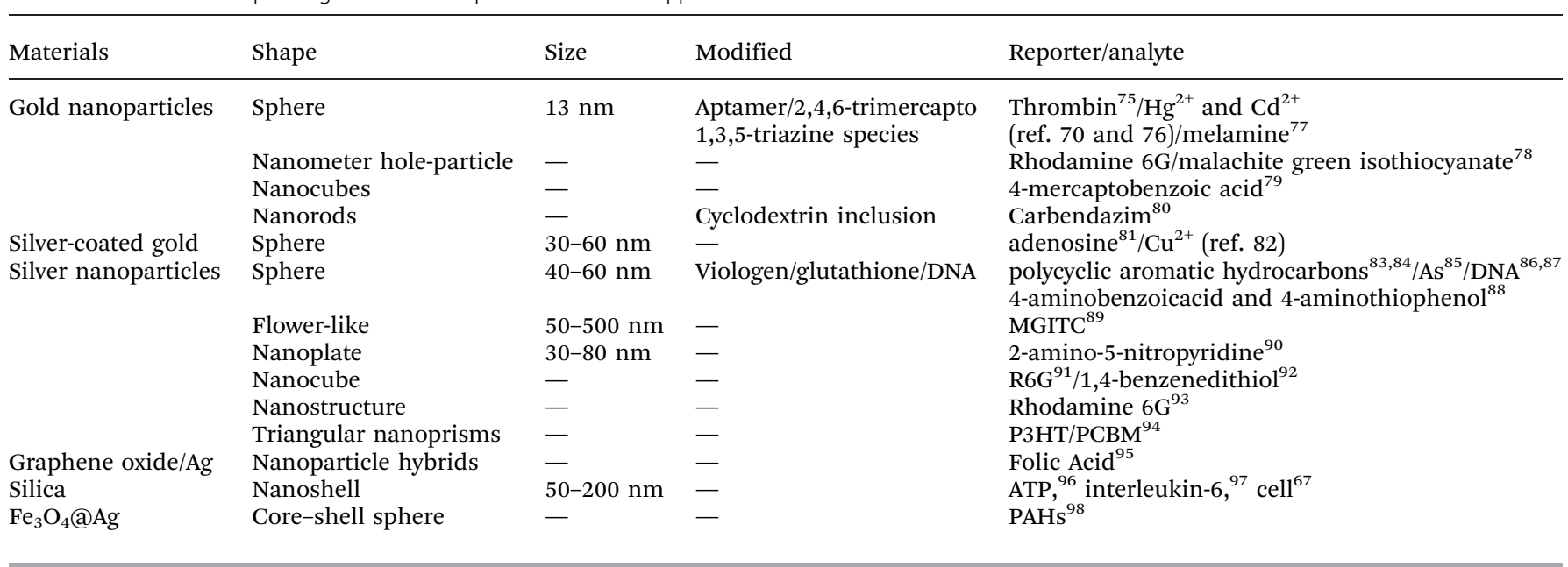

consistently formed at a specific location, namely the entrance of the nanochannel. As a result, the detection limit of SERS for adenine molecules was lower than $10 \mathrm{pM}$. Tong et al. ${ }^{65}$ reported SERS measurements of Ag colloids in a microfluidic circuit operated as a lab-on-a-chip device, where aggregation is achieved through optical forces generated by laser tweezers integrated into the Raman spectroscopy set-up. Laser tweezers are capable of trapping metal nanoparticles through field gradient forces in the direction towards the beam-waist of a tightly focused laser beam, where additional scattering and absorption forces tend to push the particles along the optical axis. In addition to these single particle forces, the laser field also generates attractive inter-particle optical forces that will cause colloidal aggregation. Therefore, the application of the laser tweezers to a stream of metal colloids thus results in an accumulation and subsequent aggregation of nanoparticles at the laser tweezers focus. The combination of optical tweezers and microfluidic technologies paves the way for novel lab-on-achip based plasmonic chemo/biosensors and overcomes two important drawbacks of standard SERS sensing schemes; i.e., the renewal of nanofabricated metallic substrates and the difficulty of avoiding uncontrolled aggregation in metal colloids.

\subsection{Metal nanocolloids}

Using the inelastic light scattering of noble metal spherical colloidal nanoparticles, SERS opens up a new application era of Raman spectroscopy because these kinds of colloidal nanoparticles have shown promise in overcoming the low sensitivity problem inherent in conventional Raman spectroscopy. ${ }^{3,10,66}$ Sphere nanoparticles as the simplest nanostructure were studied much earlier. However, the properties of NPs have a great relationship with the nature of their size and shape of noble metal nanostructures and this simple structure is far from meeting the needs of scientific investigation. To design the characteristics of nanoparticles, understanding some routine rules is very important. Firstly, the nanoparticles should provide large SERS enhancement factors and be combined with easy preparation protocol. The substrate should not be prone to environmental interference.
Secondly, to obtain good reproducibility, the metallic nanoparticles should be put into regular or highly periodic order. Finally, good biocompatibility and long-term stability are also critical when the research concerns the investigation of biological samples. ${ }^{67,68}$ Therefore, in the past decades, people found that gold and silver nanoparticles demonstrated excellent features compared with other particles. In addition, a lot of successful synthesis methods to produce different and various forms of metallic nanostructures, including sphere, cube, rod and hollow shapes, have been proposed. Additionally, for SERS enhancing technology, silver and gold nanoparticles are the most widely used among various metal colloids. Table 1 shows different kinds of shapes for gold/silver nanoparticles and some of their applications. Due to the dimensions on the nanoscale, the nanoparticles have exhibited fascinating optical properties and applications for colorimetric detection field. ${ }^{69-72}$ Gold colloidal particles have advantages including long-term stability, easily controllable size distribution, and high homogeneity. However, the Raman enhancement factor for the gold nanoparticles is $10^{2}-10^{3}$ times lower than for silver particles. Thus, despite several disadvantages, such as instability resulting from limited solubility and aggregation properties, silver nanocolloids are more widely used than gold nanocolloids.

The most popular method for preparing silver nanocolloids is the reduction of silver cations reported by Lee and Meisel. ${ }^{73}$ Silver nanocolloids have also been prepared more recently by the method reported by Leopold and Lendl. ${ }^{74}$ The advantages of the latter method are its fast preparation at room temperature and its immediate applicability for SERS. Sometimes the Raman-active substrates can be prepared by using different noble metals such as bimetallic nanoparticles (silver mixed with gold: silver-coated gold and gold-coated silver). We named them functional nanocolloids based on their fundamental characteristics. This bimetallic nanoparticle overcomes the disadvantages of both gold and silver nanoparticles mentioned above or increases the bio-compatibility. The characteristic plasmon bands of gold and silver nanocolloids can be easily identified using UV/Vis spectroscopy. Typical nanocolloids show characteristic plasmon 
bands ranging from 380 to $440 \mathrm{~nm}$ for silver and from 510 to $540 \mathrm{~nm}$ for gold, respectively. Particle size and morphological properties of metal nanoparticles can be obtained by using electronic microscopes such as TEM and AFM, etc. The information about size distributions of metal nanoparticles can be attained by using the dynamic light scattering technique.

Remarkable aspects of the SERS technique have motivated research to design new Raman-active substrates. To date, spherical colloidal nanoparticles are the most widely used as optical-enhancing substrates. However, current SERS application technology, especially for cellular imaging or biomedical diagnostics, still has a strong need for improvement in its optical nanoprobes in regard to sensitivity, selectivity, optical stability, and special localization. For this purpose, the nanoparticles such as core-shell-type nanoparticles including gold nanoshells, ${ }^{99-102}$ nanocrescents, ${ }^{103}$ and hollow gold nanospheres ${ }^{104-107}$ have been synthesized, and their applicability as effective SERS probes has been tested. ${ }^{77,85}$ The different shape nanoparticles have potential to meet the requirements of the SERS measurement. More importantly, gold nanorods (GNRs) have been introduced as powerful imaging probes for biomedical and biological applications. ${ }^{108-116}$

GNRs have two surface plasmon bands, a short-wavelength band around $520 \mathrm{~nm}$ and a long-wavelength band in the nearinfrared region. ${ }^{117-119}$ The SERS intensity is highly dependent on the maximum absorption peak of the SERS substrate (Au or $\mathrm{Ag}$ nanoparticles) and the excitation-laser wavelength. When the excitation-laser wavelength matches the optical absorption of nanoparticles and Raman reporters, then surface-enhanced resonance Raman scattering occurs, and the enhancement factor may be further enhanced 100 times. As a result, GNRs absorb and scatter electromagnetic radiation more strongly than gold nanospheres because of the enhanced surface electric field upon surface plasmon excitation. Furthermore, the GNR aggregates also show stronger SERS enhancement effects than gold nanosphere aggregates because of the increase in density of junction points caused by their large surface areas. ${ }^{120}$

There are two ways for realizing SERS on microfluidic devices. One is immobilized silver/gold on the substrate by lithographic techniques which can adsorb with the sample flow and generate the surface Raman signal, and another one depends on the continuous gold/silver particle flow mixing with reagent flow in a controlled manner. Liu $^{121}$ and Kho ${ }^{122}$ fabricated nanowell-based silver substrates and periodic active nanostructure to get SERS signal. Dluhy ${ }^{123}$ used electron beam evaporation to deposit silver arrays. Sans developed a laser electro-dispersion technique employed to deposit gold nanoparticles onto Si surfaces. ${ }^{124}$ Femtosecond laser as a promising technique that can interact with a target with ultra-fast speed and extremely high resolution also has been applied to Raman investigation. As shown in Fig. 3, Xu, Zhou and Wang et al. reported facile approaches for the flexible integration of high efficiency surface enhanced Raman scattering (SERS) monitors in a continuous microfluidic channel via femtosecond laser micro-nanofabrication. ${ }^{125-128}$ The silver substrate could be shaped into designed patterns, and could be precisely located
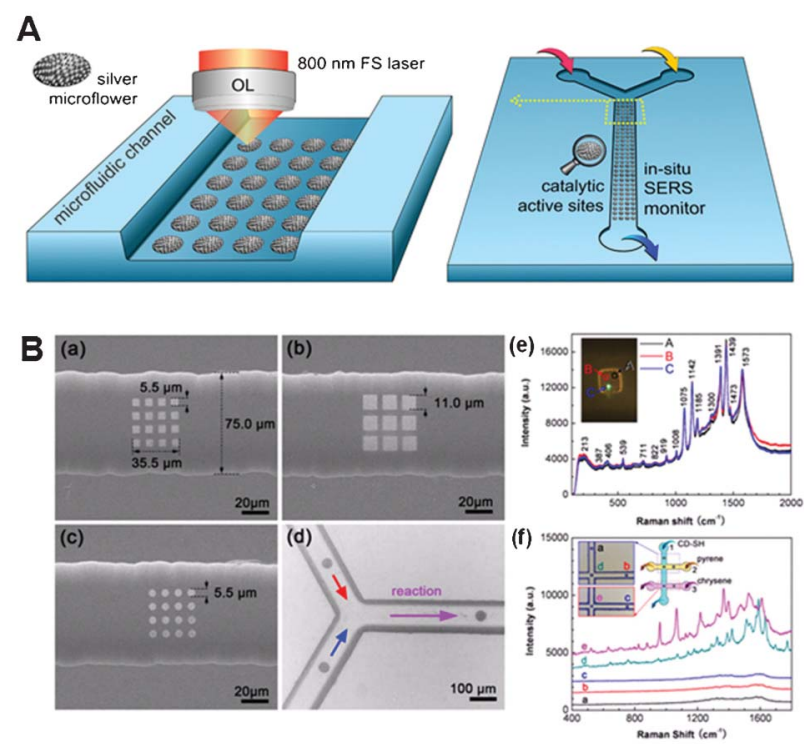

Fig. 3 (A) Scheme for laser fabrication of silver microflower arrays by femtosecond laser direct writing inside a microfluidic channel. ${ }^{126}$ Copyright 2012 Royal Society of Chemistry. (B) SEM images of silver SERS substrates integrated in a microfluidic channel with different patterns and sizes. (a) $4 \times 4$, $5.5 \mu \mathrm{m}$ square arrays; (b) $3 \times 3,11.0 \mu \mathrm{m}$ square arrays; (c) $4 \times 4$ circles arrays with diameter of $5.5 \mu \mathrm{m}$; (d) silver SERS substrates at different positions of the microchannel; (e) Raman spectra of $p$-aminothiophenol ( $p$-ATP) measured at different positions of the silver SERS substrate. Inset is the optical microscopic image of the silver SERS substrate and different measure positions; (f) Raman spectra measured on silver SERS substrates at different positions of the microchannel (a-e). Inset is the scheme of the experiments and optical microscopy image of the microchannel with silver SERS substrates. ${ }^{129}$ Copyright 2011 Royal Society of Chemistry.

at the desired position of the microchannel bed, providing the feasibility for real-time detection. ${ }^{129}$ However, the static adsorption affects the stability of the signal and the enhancement factor is not high in this manner and the high cost of equipment is a major limitation. Therefore, more people tend to choose the second way ${ }^{47,53,64}$ and individual nanoparticles will give rise to surface enhancement under the appropriate conditions. ${ }^{130}$ Maximum signal intensity can be obtained by aggregating the particles in a controlled manner to turn on a larger surface enhancement due to the increased electric field in the interstices between the nanoparticles. According to SERS data reported by Kneipp et al., ${ }^{2}$ the SERS enhancement factor for silver nanoclusters at $830 \mathrm{~nm}$ excitation were found to be seven orders of magnitude higher than that of isolated silver particles. Thus, it is very important to control the formation of nanoaggregates to obtain a maximum enhancement. Aggregation agents, such as chloride, nitrate, perchlorate, polylysine, and spermine, are generally used for signal enhancement. ${ }^{7}$ Partial aggregation of metal nanoparticles can be induced by controlling the concentration of aggregation agents.

\subsection{Instrumental}

The instrumental setup of the Raman microscope is important for the SERS detection to succeed in microfluidic applications. 
As has been discussed in detail previously, ${ }^{7,9}$ the confocal Raman microscopic technique makes it possible to investigate small samples in a narrow microfluidic channel, where a twoslit confocal arrangement was used to reduce the background Raman scattering from the unfocused laser beams. It is wellknown that PDMS is a widely used polymeric material for the design of microfluidic channels because it has favorable optical properties and exhibits an interfacial adhesion that assists bonding. However, PDMS is a Raman active polymer, which has its own Raman scattering signal. Therefore, the Raman signals from the PDMS channel have to be effectively removed to obtain real Raman signals. The confocal technique is utilized to solve this problem. In the confocal Raman microscopic technique, the adjustable slit is placed in the back image plane of the microscope's objective lens to block any light from the outside. The confocal slit is designed to collect only the laser-induced signal from a distinct focal volume within the diffraction limit. ${ }^{131}$ Though the size of the channel is very tiny, confocal technique can help people to distinguish the signal obtained from the small amounts of chemical species apart from the signal originating from the surrounding PDMS material easily. Moreover, the confocal optical arrangements can find convenient applications not only for SERS microfluidic chip detection, but also for other similar microtubules by focusing the laser on the confocal Raman stage.

In order to meet the requirements of real-time analytical technologies to be applied in environmental monitoring, forensic analysis and homeland security, portable Raman spectrometers obviously become good candidates. Combination of the portable Raman microscopes with labon-a-chip, to a certain extent, provides future ideal implements for the quantitative measurement of trace analytes. Recently, several different types of portable Raman systems have been developed, and are commercially available now. ${ }^{132,133}$ However, some essential problems of portable Raman systems must be resolved to satisfy the need of fast and accurate analysis. Raman scattering is an extremely inefficient process with a low scattering cross section. The detection sensitivity of portable Raman systems is inferior to that of large Raman spectroscopic systems, because all the spectroscopic components, including the laser source, monochromator and detector, are miniaturized. As we know, quantitative trace analysis using SERS is very difficult, because it is awkward to control the experimental conditions, such as the degree of aggregation, the particle size of the metal colloids and the inhomogeneous distribution of molecules on the metal surface. To solve this problem, Choo's group ${ }^{27,134,135}$ successfully combined SERS detection by using metal nanoparticles with a lab-on-a-chip technique. Compared with conventional SERS detection under static conditions, the SERS detection in a microfluidic channel has some advantages. A highly accurate quantitative analysis is possible when a continuous flow and homogeneous mixing conditions between the analytes and metal nanocolloids are maintained in a microfluidic channel. ${ }^{134,135}$ In addition, SERS-microfluidic systems combined with optical fiber sensors ${ }^{136}$ or optrodes ${ }^{137}$ may be a potential trend in the future because they offer better portability and lower signal background.
Briefly, the development of Raman instrumentation has been relatively rapid in recent years. This may be attributed to two aspects. On the one hand, with the development of laser technology, ultrafast laser-based nonlinear Raman spectroscopy has become increasingly mature. This sophisticated technology requires expensive equipment and originally only a very few units could engage. However, this situation no longer exists, and the price of the instrument is becoming relatively low. Confocal Raman spectroscopy has reached a relatively mature and stable stage. The second important aspect is the rapid development of nanotechnology. The nanostructurebased surface-enhanced Raman spectroscopy has made considerable progress in ultra-high sensitivity detection, which is especially significant for portable Raman equipment. Because of the demand for portable and low-cost equipment, the complicated and extensive optical system is not suitable, and nanotechnology paves the way and plays a very important role for implementing high sensitivity detection. Depending on Raman's attractive features of noninvasive analysis, and good spectral resolution, it can greatly satisfy the increasing requirements of resource-limited settings, homeland security and on-site detection.

\section{Applications}

\subsection{Biological and biomedical}

As a physicochemical technique, Raman spectroscopy could generate a spectroscopic fingerprint from the DNA, protein or cell and provides their quantitative and qualitative information without damaging the microbial sample. ${ }^{138,139}$ These advantages showed tremendous potential for bio/chemical molecular analysis at the trace and even single molecule level. The microfluidic system provides a highly defined space, and can easily and accurately handle a variety of biological reactions. Combining the remarkable features of lab-on-a-chip technology with Raman spectroscopy provides a promising platform for synergistically integrating the variety of implemented functions with flexible sample control, ${ }^{140-142}$ and the sensitive, rapid and noninvasive detecting results have attracted much attention in recent years. Choo and co-workers designed a gradient optofluidic microfluidic device and applied it for a fast and sensitive Raman detection of immunoassay reactions. ${ }^{143,144}$ By using Kwang's novel gradient method, Lee developed a programmable and fully automatic gold array-embedded gradient optofluidic chip for Raman ELISA analysis. ${ }^{35}$ The microfluidic channel matches the size of bacteria very well which is conducive to the cultivation of the bacteria or cell ${ }^{141,145-147}$ that offers the possibility of system integration issues for bio/chemical molecular analysis at the trace and even single molecule level. ${ }^{3,148,149}$ Walter et al. ${ }^{150}$ presented a fast, reliable and specific test of bacteria by means of SERS in a microfluidic device (Fig. 4(a)). They proved the vibrational spectroscopy was feasible for the validation of bacteria by analyzing the model system $E$. coli in their microfluidic devices. The specific spectra were reproducible. By using microfluidic flow cell, the scytonemin as the sheath pigment unique to cyanobacteria 


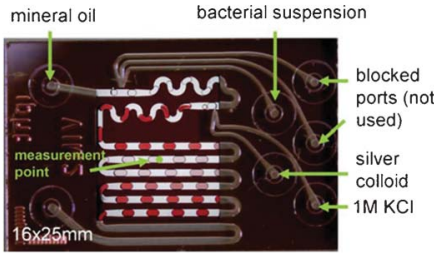

(a)

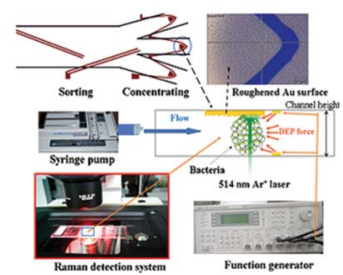

(b)
Fig. 4 (a) The microfluidic device for high specific and reliable discrimination of bacteria by SERS. ${ }^{150}$ Copyright 2011 Royal Society of Chemistry. (b) System configuration of the setup for dielectrophoretic combined with SERS detection. ${ }^{152}$ Copyright 2010 American Institute of Physics.

was detected by means of SERS. ${ }^{151}$ As illustrated in Fig. 4(b), Cheng $^{152}$ present the method by in situ surface-enhanced Raman scattering (SERS) to make an analysis of bacteria on a microfluidic platform and bacteria could be continuously sorted and concentrated via three-dimensional dielectrophoresis. Through SERS spectral analysis, the Gram positive and Gram negative bacteria could be easily effectively distinguished in several minutes.

Genomics offers a comprehensive view of the complete genetic makeup of an organism. Individual sequence variations, as manifested by single nucleotide polymorphisms (SNPs), can provide insight into the basis for a large number of phenotypes and diseases including cancer. The ability to rapidly screen for SNPs will have a profound impact on numerous applications, most notably in personalized medicine. Huh et al. ${ }^{153}$ demonstrated a new approach to SNP detection through the application of SERS to the ligase detection reaction. In order to demonstrate the feasibility of antibody-conjugated GNRs for the highly sensitive targeting and imaging of specific cancer markers expressed on the surface membrane of cancer cells, GNRs with different aspect ratios were synthesized, and their SERS enhancement capability was evaluated. ${ }^{154}$ Target-oriented antibodies were immobilized on the surface of GNRs by using the layer-bylayer (LBL) deposition method. A SERS-active Raman reporter, 4-mercaptopyridine (MP) and anti-rabbit IgG were conjugated by the LBL deposition method. MCF7 cells, over expressing human epithermal growth factor (HER2) markers on their surface membrane, were used as optical imaging targets. HER2 is a clinically significant molecular marker of breast cancer. ${ }^{154,155}$

\subsection{Environmental}

Real-time analytical technologies are currently in demand for their applications in environmental monitoring, forensic science and homeland security. In particular, the rapid and accurate identification of hazardous materials in the field is important for prompt prevention of their spreading. A portable Raman spectrometer is a good candidate for the real-time analysis of hazardous materials in the field because it is possible to analyse a sample rapidly, as well as detect its signal non-destructively. An integrated real-time sensing system that uses a portable Raman spectrometer and a micropillar array chip has been developed for field analysis. ${ }^{27}$ Two hazardous materials, DPA and MB, were quantitatively analyzed using an integrated portable Raman sensor system. The observed limits of detection were estimated to be 200 and $500 \mathrm{ppb}$, respectively. The proposed analytical method, using a micropillar array PDMS chip and a portable SERS system, offers a rapid and reproducible trace detection capability for hazardous materials in the field.

The use of SERS detection for the highly sensitive trace analysis of mercury (II) ions in water within a droplet-based microfluidic system has been demonstrated recently. ${ }^{48}$ The novel combination of droplet-based microfluidics with SERS detection provides both fast and sensitive detection of mercury (II) ions in water. Choi et al. ${ }^{156}$ have presented a CD-based microfluidic device for high throughput SERS detections (Fig. 5(a)), and this platform could be effectively used for label-free environmental and biomolecular analysis.

Recently, Whiteside's group proposed a promising low-cost paper microfluidic technique, ${ }^{157,158}$ that has great advantages including inexpensive price, prominent device portability and low sample consumption. Hence, it had been applied to the ELISA, ${ }^{159}$ Electrochemical and chemiluminescence sen- (a)

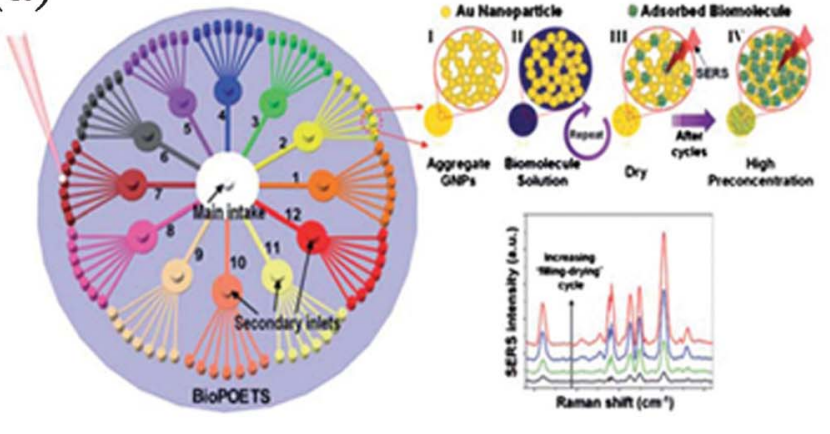

(b)

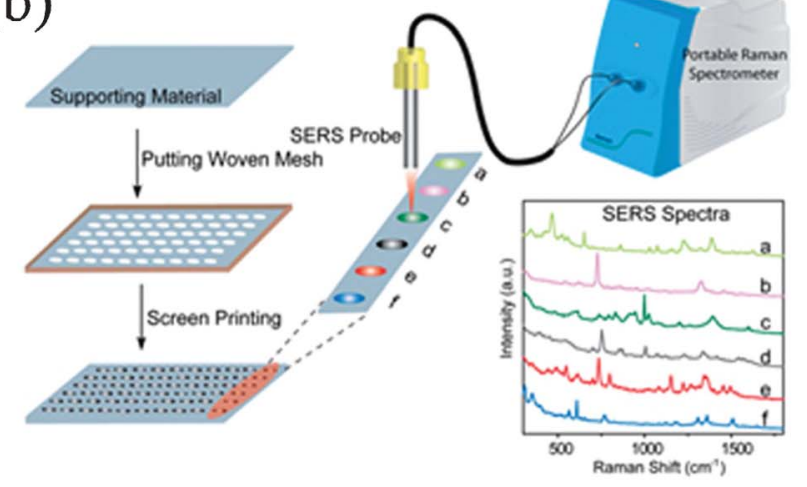

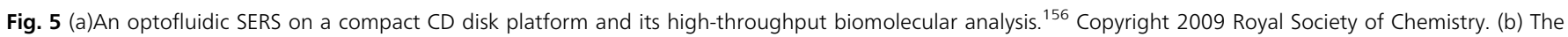

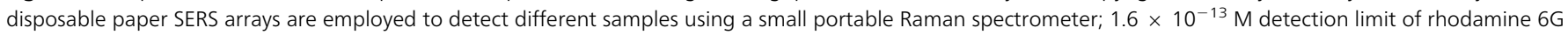
could be achieved. ${ }^{166}$ Copyright 2012 Royal Society of Chemistry. 
sing, ${ }^{160-163}$ point-of-care testing, ${ }^{163,164}$ and the SERS field. ${ }^{165,166}$ As shown in Fig. 5(b), an inexpensive and facile fabrication of SERS arrays was illustrated, and the printed paper has good SERS reproducibility and can be disposed of by burning without any environmental pollution.

\subsection{Separation detection}

Separation and detection of different proteins is fundamental for the understanding of biological systems. Gel-electrophoresis and liquid chromatography are popular methods for protein separation. The disadvantage is their inconvenient sample preparations which possibly change the structure and functionality of the protein. Therefore, the results of further analysis may not represent the native conformation of the protein. However, the SERS-based microfluidic system is a promising way to detect biomolecules. ${ }^{167,168}$ Becker et al. ${ }^{169}$ presented a new combination of free-flow electrophoresis (FFE) microfluidic chip and SERS to detect a label-free optical sample. Morris et al. ${ }^{170}$ have carried out conventional Raman after a separation of pesticides. Despite the high quality of this work, the low scattering cross-section of most molecules for conventional Raman severely limits the breadth of analytes that can be sensitively detected in such a system.

Smith et al. ${ }^{171,172}$ have achieved SERS detection of dye molecules with colloidal substrates reduced on-chip. Farquharson et al. ${ }^{173}$ have shown some spatial differentiation between SERS spectra within a channel fabricated of their Raman-enhancing vial material; however, the separation channel's cross-sectional area is too large to effect any useful separations. These works are not full integrations of electrophoretic separation with SERS detection where the signal enhancing substrate is built directly into the architecture of the microfluidic device. Connatser et al. ${ }^{174}$ have demonstrated the feasibility of using microfluidic separation devices with integrated regions of metal-polymer nanocomposites for SERS detection of separated analytes. Connatser et al. ${ }^{175}$ have presented integrating SERS-based detection technique directly into a device for microfluidic electrophoretic separation chip, where the separated electrophoretic bands are structurally descriptively identified. This method represents a novel detection mode for microfluidics that addresses an inherent shortcoming of CE-style separations.

\section{Conclusions}

In this mini-review, recent advances of the SERS-based microfluidic systems and their applications in various chemical and biological analyses have been discussed. Combination of the SERS technique with lab-on-a-chip provides reproducible results for the quantitative measurement of analytes at low concentration levels, which is a vital breakthrough for SERS to be a quantitative technique because it overcomes the difficulties related to the experimental conditions, such as the degree of aggregation, the different sizes of metal colloids, and inhomogeneous distributions of molecules on the metal surface. Various relevant techniques of SERS-based microfluidic systems such as the microfluidic mixer for efficient mixing, the different shaped nanoparticles, and prominent applications of SERS detection in microfluidic systems for environmental trace analyses, biomedical and biological assays and separation detection, have been discussed. Finally, most present Raman systems are composed of bulky optical components, such as an optical microscope, laser, monochromator, and detector, external to the microfluidic chip. A portable miniaturized Raman system has made substantial progress. With extensive on-going experimental and theoretical efforts, more techniques will promote the development of this area, such as advances in nanofabrication and self-assembly, high performance low-cost laser, and embedded optical fiber system. The interests in applying SERS combining lab-on-a-chip technique in the area of environmental monitoring, homeland security and bio-analysis will be constantly growing in the near future. We believe that a large number of the practical applications of SERS will be increasingly important in the next few decades.

\section{Acknowledgements}

The authors are thankful for the support of the National Natural Science Foundation of China (grant numbers 20875058 and 21205131) and the Science and Technology Development Plan of Yantai (2011071).

\section{Notes and References}

1 D. Cialla, A. Maerz, R. Boehme, F. Theil, K. Weber, M. Schmitt and J. Popp, Anal. Bioanal. Chem., 2012, 403, 27-54.

2 J. Kneipp, H. Kneipp and K. Kneipp, Proc. Natl. Acad. Sci. U. S. A., 2006, 103, 17149-17153.

3 S. M. Nie and S. R. Emery, Science, 1997, 275, 1102-1106.

4 E. C. Le Ru, E. Blackie, M. Meyer and P. G. Etchegoin, J. Phys. Chem. C, 2007, 111, 13794-13803.

5 G. T. Taylor, S. K. Sharma and K. Mohanan, Appl. Spectrosc., 1990, 44, 635-640.

6 J. J. Laserna, Anal. Chim. Acta, 1993, 283, 607-622.

7 L. Chen and J. Choo, Electrophoresis, 2008, 29, 1815-1828.

8 A. Q. Liu, H. J. Huang, L. K. Chin, Y. F. Yu and X. C. Li, Anal. Bioanal. Chem., 2008, 391, 2443-2452.

9 C. Lim, J. Hong, B. G. Chung, A. J. deMello and J. Choo, Analyst, 2010, 135, 837-844.

10 M. Fan, G. F. S. Andrade and A. G. Brolo, Anal. Chim. Acta, 2011, 693, 7-25.

11 J. C. Hulteen and R. P. Vanduyne, J. Vac. Sci. Technol., A, 1995, 13, 1553-1558.

12 C. Wulfman, N. Djaker, N. Dupont, D. Ruse, M. Sadoun and M. L. la Chapelle, J. Am. Ceram. Soc., 2012, 95, 2347-2351.

13 F. Toderas, M. Baia, L. Baia and S. Astilean, Nanotechnology, 2007, 18.

14 O. Seitz, M. M. Chehimi, E. Cabet-Deliry, S. Truong, N. Felidj, C. Perruchot, S. J. Greaves and J. F. Watts, Colloids Surf., A, 2003, 218, 225-239. 
15 T. M. Squires and S. R. Quake, Rev. Mod. Phys., 2005, 77, 977-1026.

16 G. M. Whitesides, Nature, 2006, 442, 368-373.

17 B. Li, L. Jiang, Q. Wang, J. Oin and B. Lin, Electrophoresis, 2008, 29, 4906-4913.

18 D. J. Harrison, K. Fluri, K. Seiler, Z. H. Fan, C. S. Effenhauser and A. Manz, Science, 1993, 261, 895-897.

19 L. X. Chen, J. P. Ma, F. Tan and Y. F. Guan, Sens. Actuators, $B, 2003,88,260-265$.

20 L. Chen, S. Lee, J. Choo and E. K. Lee, J. Micromech. Microeng., 2008, 18.

21 L. Chen, J. Choot and B. Yan, Expert Opin. Drug Delivery, 2007, 4, 119-129.

22 The Reynolds number is defined as $R e=\rho U L / \eta$ in which $U$ is a characteristic fluid velocity (for example the mean velocity in a channel).

23 The Péclet number is defined as $P e=U L / D, L$ is the characteristic length scale, and $D$ is the typical diffusion coefficient. $P e$ indicates the relative importance of convection to diffusion, and in channel flow its value can be interpreted as the number of channel widths the fluid needs to travel in the channel direction to get complete mixing by diffusion. For water-like liquids, typical viscosities and densities are $\eta=1 \mathrm{mPa}$ and $\rho=$ $1000 \mathrm{~kg} \mathrm{~m}^{-3}$, and a typical diffusion coefficient of small proteins is $D=1 \times 10^{-10} \mathrm{~m}^{2} \mathrm{~s}^{-1}$, so that, typically, the ratio of Péclet number and Reynolds number is 10 000. In microfluidic systems, this leads to Péclet numbers of the order of 10000 or less.

24 N. T. Nguyen and Z. G. Wu, J. Micromech.Microeng., 2005, 15, R1-R16.

25 F. G. Bessoth, A. J. deMello and A. Manz, Anal. Commun., 1999, 36, 213-215.

26 R. H. Liu, M. A. Stremler, K. V. Sharp, M. G. Olsen, J. G. Santiago, R. J. Adrian, H. Aref and D. J. Beebe, J. Microelectromech. Syst., 2000, 9, 190-197.

27 L. X. Quang, C. Lim, G. H. Seong, J. Choo, K. J. Do and S.K. Yoo, Lab Chip, 2008, 8, 2214-2219.

28 A. D. Stroock, S. K. W. Dertinger, A. Ajdari, I. Mezic, H. A. Stone and G. M. Whitesides, Science, 2002, 295, 647-651.

29 S. H. Wong, P. Bryant, M. Ward and C. Wharton, Sens. Actuators, B, 2003, 95, 414-424.

30 V. Mengeaud, J. Josserand and H. H. Girault, Anal. Chem., 2002, 74, 4279-4286.

31 L. Chen, G. Wang, C. Lim, G. H. Seong, J. Choo, E. K. Lee, S. H. Kang and J. M. Song, Microfluid. Nanofluid., 2009, 7, 267-273.

32 A. A. S. Bhagat, E. T. K. Peterson and I. Papautsky, J. Micromech. Microeng., 2007, 17, 1017-1024.

33 K. K. Unger, R. Skudas and M. M. Schulte, J. Chromatogr., A, 2008, 1184, 393-415.

34 K. Lee, C. Kim, B. Ahn, R. Panchapakesan, A. R. Full, L. Nordee, J. Y. Kang and K. W. Oh, Lab Chip, 2009, 9, 709-717.

35 M. Lee, K. Lee, K. H. Kim, K. W. Oh and J. Choo, Lab Chip, 2012, 12, 3720-3727.

36 N. Choi, K. Lee, D. W. Lim, E. K. Lee, S.-I. Chang, K. W. Oh and J. Choo, Lab Chip, 2012, 12, 5160-5167.

37 H. Song, J. D. Tice and R. F. Ismagilov, Angew. Chem., Int. Ed., 2003, 42, 768-772.
38 A. Huebner, M. Srisa-Art, D. Holt, C. Abell, F. Hollfelder, A. J. deMello and J. B. Edel, Chem. Commun., 2007, 1218-1220.

39 M. Srisa-Art, A. J. deMello and J. B. Edel, Anal. Chem., 2007, 79, 6682-6689.

40 M. Srisa-Art, A. J. deMello and J. B. Edel, Phys. Rev. Lett., 2008, 101.

41 A. Huebner, L. F. Olguin, D. Bratton, G. Whyte, W. T. S. Huck, A. J. de Mello, J. B. Edel, C. Abell and F. Hollfelder, Anal. Chem., 2008, 80, 3890-3896.

42 A. Huebner, S. Sharma, M. Srisa-Art, F. Hollfelder, J. B. Edel and A. J. deMello, Lab Chip, 2008, 8, 1244-1254.

43 S. Zeng, B. Li, X. O. Su, J. Qin and B. Lin, Lab Chip, 2009, 9, 1340-1343.

44 X. Niu, S. Gulati, J. B. Edel and A. J. deMello, Lab Chip, 2008, 8, 1837-1841.

45 D. R. Link, E. Grasland-Mongrain, A. Duri, F. Sarrazin, Z. D. Cheng, G. Cristobal, M. Marquez and D. A. Weitz, Angew. Chem., Int. Ed., 2006, 45, 2556-2560.

46 F. Courtois, L. F. Olguin, G. Whyte, D. Bratton, W. T. S. Huck, C. Abell and F. Hollfelder, ChemBioChem, 2008, 9, 439-446.

47 M. P. Cecchini, J. Hong, C. Lim, J. Choo, T. Albrecht, A. J. deMello and J. B. Edel, Anal. Chem., 2011, 83, 3076-3081.

48 G. Wang, C. Lim, L. Chen, H. Chon, J. Choo, J. Hong and A. J. de Mello, Anal. Bioanal. Chem., 2009, 394, 1827-1832.

49 D. T. Chiu, R. M. Lorenz and G. D. M. Jeffries, Anal. Chem., 2009, 81, 5111-5118.

50 S.-Y. Teh, R. Lin, L.-H. Hung and A. P. Lee, Lab Chip, 2008, 8, 198-220.

51 K. R. Strehle, D. Cialla, P. Roesch, T. Henkel, M. Koehler and J. Popp, Anal. Chem., 2007, 79, 1542-1547.

52 K. R. Ackermann, T. Henkel and J. Popp, ChemPhysChem, 2007, 8, 2665-2670.

53 C. D. Syme, C. Martino, R. Yusvana, N. M. S. Sirimuthu and J. M. Cooper, Anal. Chem., 2012, 84, 1491-1495.

54 A. O. El Moctar, N. Aubry and J. Batton, Lab Chip, 2003, 3, 273-280.

55 N. Sasaki, T. Kitamori and H. B. Kim, Lab Chip, 2006, 6, 550-554.

56 L. H. Lu, K. S. Ryu and C. Liu, J. Microelectromech. Syst., 2002, 11, 462-469.

57 K. Sritharan, C. J. Strobl, M. F. Schneider, A. Wixforth and Z. Guttenberg, Appl. Phys. Lett., 2006, 88.

58 J. den Toonder, F. Bos, D. Broer, L. Filippini, M. Gillies, J. de Goede, T. Mol, M. Reijme, W. Talen, H. Wilderbeek, V. Khatavkar and P. Anderson, Lab Chip, 2008, 8, 533-541.

59 I. Glasgow and N. Aubry, Lab Chip, 2003, 3, 114-120.

60 D. J. Kim, H. J. Oh, T. H. Park, J. B. Choo and S. H. Lee, Analyst, 2005, 130, 293-298.

61 L. Chen, S. Lee, M. Lee, C. Lim, J. Choo, J. Y. Park, S. Lee, S.-W. Joo, K.-H. Lee and Y.-W. Choi, Biosens. Bioelectron., 2008, 23, 1878-1882.

62 S. Lee, J. Choi, L. Chen, B. Park, J. B. Kyong, G. H. Seong, J. Choo, Y. Lee, K.-H. Shin, E. K. Lee, S.-W. Joo and K.H. Lee, Anal. Chim. Acta, 2007, 590, 139-144.

63 J. Jung, L. Chen, S. Lee, S. Kim, G. H. Seong, J. Choo, E. K. Lee, C.-H. Oh and S. Lee, Anal. Bioanal. Chem., 2007, 387, 2609-2615. 
64 M. Wang, N. Jing, I. H. Chou, G. L. Cote and J. Kameoka, Lab Chip, 2007, 7, 630-632.

65 L. Tong, M. Righini, M. Ujue Gonzalez, R. Quidant and M. Kall, Lab Chip, 2009, 9, 193-195.

66 C. R. Yonzon, C. L. Haynes, X. Y. Zhang, J. T. Walsh and R. P. Van Duyne, Anal. Chem., 2004, 76, 78-85.

67 Y. Wang, L. Chen and P. Liu, Chem.-Eur. J., 2012, 18, 5935-5943.

68 Y. Wang, B. Yan and L. Chen, Chemical Reviews, 2013, 113, 1391-1428.

69 J. W. Liu and Y. Lu, J. Am. Chem. Soc., 2003, 125, 6642-6643.

70 T. Lou, Z. Chen, Y. Wang and L. Chen, ACS Appl. Mater. Interfaces, 2011, 3, 1568-1573.

71 G. Wang, Z. Chen, W. Wang, B. Yan and L. Chen, Analyst, 2011, 136, 174-178.

72 L. Chen, T. Lou, C. Yu, Q. Kang and L. Chen, Analyst, 2011, 136, 4770-4773.

73 P. C. Lee and D. Meisel, J. Phys. Chem., 1982, 86, 3391-3395.

74 N. Leopold and B. Lendl, J. Phys. Chem. B, 2003, 107, 5723-5727.

75 H. Cho, B. R. Baker, S. Wachsmann-Hogiu, C. V. Pagba, T. A. Laurence, S. M. Lane, L. P. Lee and J. B. H. Tok, Nano Lett., 2008, 8, 4386-4390.

76 V. M. Zamarion, R. A. Timm, K. Araki and H. E. Toma, Inorg. Chem., 2008, 47, 2934-2936.

77 T. Lou, Y. Wang, J. Li, H. Peng, H. Xiong and L. Chen, Anal. Bioanal. Chem., 2011, 401, 333-338.

78 H. Wei, U. Hakanson, Z. Yang, F. Hook and H. Xu, Small, 2008, 4, 1296-1300.

79 P. N. Sisco and C. J. Murphy, J. Phys. Chem. A, 2009, 113, 3973-3978.

80 A. D. Strickland and C. A. Batt, Anal. Chem., 2009, 81, 2895-2903.

81 J.-W. Chen, X.-P. Liu, K.-J. Feng, Y. Liang, J.-H. Jiang, G.L. Shen and R.-Q. Yu, Biosens. Bioelectron., 2008, 24, 66-71.

82 T. Lou, L. Chen, Z. Chen, Y. Wang, L. Chen and J. Li, ACS Appl. Mater. Interfaces, 2011, 3, 4215-4220.

83 L. Guerrini, J. V. Garcia-Ramos, C. Domingo and S. Sanchez-Cortes, Anal. Chem., 2009, 81, 953-960.

84 L. Guerrini, J. V. Garcia-Ramos, C. Domingo and S. Sanchez-Cortes, Anal. Chem., 2009, 81, 1418-1425.

$85 \mathrm{~J}$. Li, L. Chen, T. Lou and Y. Wang, ACS Appl. Mater. Interfaces, 2011, 3, 3936-3941.

86 Z. Zhang, Y. Wen, Y. Ma, J. Luo, L. Jiang and Y. Song, Chem. Commun., 2011, 47, 7407-7409.

87 Y. W. C. Cao, R. C. Jin and C. A. Mirkin, Science, 2002, 297, 1536-1540.

88 W.-L. Zhai, D.-W. Li, L.-L. Qu, J. S. Fossey and Y.-T. Long, Nanoscale, 2012, 4, 137-142.

89 J. H. Bang and K. S. Suslick, Adv. Mater., 2009, 21, 3186.

90 A. C. Sant'Ana, T. C. R. Rocha, P. S. Santos, D. Zanchet and M. L. A. Temperini, J. Raman Spectrosc., 2009, 40, 183-190.

91 M. Rycenga, X. Xia, C. H. Moran, F. Zhou, D. Qin, Z.-Y. Li and Y. Xia, Angew. Chem., Int. Ed., 2011, 50, 5473-5477.

92 J. M. McLellan, Z.-Y. Li, A. R. Siekkinen and Y. Xia, Nano Lett., 2007, 7, 1013-1017.
93 C.-C. Chang, K.-H. Yang, Y.-C. Liu and C.-C. Yu, Anal. Chim. Acta, 2012, 709, 91-97.

94 M. Stavytska-Barba, M. Salvador, A. Kulkarni, D. S. Ginger and A. M. Kelley, J. Phys. Chem. C, 2011, 115, 20788-20794.

95 W. Ren, Y. Fang and E. Wang, ACS Nano, 2011, 5, 6425-6433.

96 M. Li, J. Zhang, S. Suri, L. J. Sooter, D. Ma and N. Wu, Anal. Chem., 2012, 84, 2837-2842.

97 Y. Wang, M. Salehi, M. Schuetz, K. Rudi and S. Schluecker, Analyst, 2013, 138, 1764-1771.

98 J. Du and C. Jing, J. Phys. Chem. C, 2011, 115, 17829-17835.

99 S. Lal, N. K. Grady, J. Kundu, C. S. Levin, J. B. Lassiter and N. J. Halas, Chem. Soc. Rev., 2008, 37, 898-911.

100 H. Wang, J. Kundu and N. J. Halas, Angew. Chem., Int. Ed., 2007, 46, 9040-9044.

101 C. Loo, A. Lowery, N. J. Halas, J. West and R. Drezek, Nano Lett., 2005, 5, 709-711.

102 S. Lee, S. Kim, J. Choo, S. Y. Shin, Y. H. Lee, H. Y. Choi, S. Ha, K. Kang and C. H. Oh, Anal. Chem., 2007, 79, 916-922.

103 Y. Lu, G. L. Liu and L. P. Lee, Nano Lett., 2005, 5, 5-9.

104 A. M. Schwartzberg, T. Y. Oshiro, J. Z. Zhang, T. Huser and C. E. Talley, Anal. Chem., 2006, 78, 4732-4736.

105 A. M. Schwartzberg, T. Y. Olson, C. E. Talley and J. Z. Zhang, J. Phys. Chem. B, 2006, 110, 19935-19944.

106 S. Lee, H. Chon, M. Lee, J. Choo, S. Y. Shin, Y. H. Lee, I. J. Rhyu, S. W. Son and C. H. Oh, Biosens. Bioelectron., 2009, 24, 2260-2263.

107 H. Chon, S. Lee, S. W. Son, C. H. Oh and J. Choo, Anal. Chem., 2009, 81, 3029-3034.

108 H. Takahashi, Y. Niidome, T. Niidome, K. Kaneko, H. Kawasaki and S. Yamada, Langmuir, 2006, 22, 2-5.

109 X. H. Huang, I. H. El-Sayed, W. Qian and M. A. El-Sayed, J. Am. Chem. Soc., 2006, 128, 2115-2120.

110 X. Huang, I. H. El-Sayed, W. Qian and M. A. El-Sayed, Nano Lett., 2007, 7, 1591-1597.

111 T. S. Hauck, A. A. Ghazani and W. C. W. Chan, Small, 2008, 4, 153-159.

112 C. Yu, L. Varghese and J. Irudayaraj, Langmuir, 2007, 23, 9114-9119.

113 C. Yu, H. Nakshatri and J. Irudayaraj, Nano Lett., 2007, 7, 2300-2306.

114 P. F. Jiao, H. Y. Zhou, L. X. Chen and B. Yan, Curr. Med. Chem., 2011, 18, 2086-2102.

115 S. Dochow, C. Krafft, U. Neugebauer, T. Bocklitz, T. Henkel, G. Mayer, J. Albert and J. Popp, Lab Chip, 2011, 11, 1484-1490.

116 A. Maerz, B. Moench, P. Roesch, M. Kiehntopf, T. Henkel and J. Popp, Anal. Bioanal. Chem., 2011, 400, 2755-2761.

117 C. J. Orendorff, L. Gearheart, N. R. Jana and C. J. Murphy, Phys. Chem. Chem. Phys., 2006, 8, 165-170.

118 B. Nikoobakht and M. A. El-Sayed, J. Phys. Chem. A, 2003, 107, 3372-3378.

119 B. Nikoobakht and M. A. El-Sayed, Chem. Mater., 2003, 15, 1957-1962.

120 S. Yun, Y.-K. Park, S. K. Kim and S. Park, Anal. Chem., 2007, 79, 8584-8589.

121 G. L. Liu and L. P. Lee, Appl. Phys. Lett., 2005, 87. 
122 K. W. Kho, K. Z. M. Qing, Z. X. Shen, I. B. Ahmad, S. S. C. Lim, S. Mhaisalkar, T. J. White, F. Watt, K. C. Soo and M. Olivo, J. Biomed. Opt., 2008, 13.

123 Y. Liu, J. Fan, Y. P. Zhao, S. Shanmukh and R. A. Dluhy, Applied Physics Letters, 2006, 89.

124 V. Sans, A. Moskalenko, K. Wilson, V. kozhevin, D. Yavsin, I. Kuzmin, S. Gurevich and A. Lapkin, Analyst, 2011, 136, 3295-3302.

125 J. Wang, Y. He, H. Xia, L.-G. Niu, R. Zhang, Q.-D. Chen, Y.L. Zhang, Y.-F. Li, S.-J. Zeng, J.-H. Qin, B.-C. Lin and H.B. Sun, Lab Chip, 2010, 10, 1993-1996.

126 B.-B. Xu, R. Zhang, X.-Q. Liu, H. Wang, Y.-L. Zhang, H.B. Jiang, L. Wang, Z.-C. Ma, J.-F. Ku, F.-S. Xiao and H.B. Sun, Chem. Commun., 2012, 48, 1680-1682.

127 B.-B. Xu, Z.-C. Ma, H. Wang, X.-Q. Liu, Y.-L. Zhang, X.L. Zhang, R. Zhang, H.-B. Jiang and H.-B. Sun, Electrophoresis, 2011, 32, 3378-3384.

128 Z. Zhou, J. Xu, Y. Liao, Y. Cheng, Z. Xu, K. Sugioka and K. Midorikawa, Opt. Commun., 2009, 282, 1370-1373.

129 B.-B. Xu, Z.-C. Ma, L. Wang, R. Zhang, L.-G. Niu, Z. Yang, Y.-L. Zhang, W.-H. Zheng, B. Zhao, Y. Xu, Q.-D. Chen, H. Xia and H.-B. Sun, Lab Chip, 2011, 11, 3347-3351.

130 K. Faulds, R. E. Littleford, D. Graham, G. Dent and W. E. Smith, Anal. Chem., 2004, 76, 592-598.

131 M. Navratil, G. A. Mabbott and E. A. Arriaga, Anal. Chem., 2006, 78, 4005-4019.

132 B. R. Wood, P. Heraud, S. Stojkovic, D. Morrison, J. Beardall and D. McNaughton, Anal. Chem., 2005, 77, 4955-4961.

133 F. Yan and T. Vo-Dinh, Sens. Actuators, B, 2007, 121, 61-66.

134 T. Park, S. Lee, G. H. Seong, J. Choo, E. K. Lee, Y. S. Kim, W. H. Ji, S. Y. Hwang, D. G. Gweon and S. Lee, Lab Chip, 2005, 5, 437-442.

135 K. Yea, S. Lee, J. B. Kyong, J. Choo, E. K. Lee, S. W. Joo and S. Lee, Analyst, 2005, 130, 1009-1011.

136 P. C. Ashok, G. P. Singh, K. M. Tan and K. Dholakia, Opt. Express, 2010, 18, 7642-7649.

137 M. Fan, P. Wang, C. Escobedo, D. Sinton and A. G. Brolo, Lab Chip, 2012, 12, 1554-1560.

138 R. M. Jarvis and R. Goodacre, Chem. Soc. Rev., 2008, 37, 931-936.

139 S. Efrima and L. Zeiri, J. Raman Spectrosc., 2009, 40, 277-288.

140 T. Thorsen, S. J. Maerkl and S. R. Quake, Science, 2002, 298, 580-584.

141 F. K. Balagadde, L. C. You, C. L. Hansen, F. H. Arnold and S. R. Quake, Science, 2005, 309, 137-140.

142 B. Li, L. Jiang, H. Xie, Y. Gao, J. Qin and B. Lin, Electrophoresis, 2009, 30, 3053-3057.

143 H. Chon, C. Lim, S.-M. Ha, Y. Ahn, E. K. Lee, S.-I. Chang, G. H. Seong and J. Choo, Anal. Chem., 2010, 82, 5290-5295.

144 M. Lee, S. Lee, J.-H. Lee, H.-W. Lim, G. H. Seong, E. K. Lee, S.-I. Chang, C. H. Oh and J. Choo, Biosens. Bioelectron., 2011, 26, 2135-2141.

145 L. Zhao, Z. Wang, S. Fan, Q. Meng, B. Li, S. Shao and Q. Wang, Biomed. Microdevices, 2010, 12, 325-332.

146 D. Liu, L. Wang, R. Zhong, B. Li, N. Ye, X. Liu and B. Lin, J. Biotechnol., 2007, 131, 286-292.

147 R. Gomez-Sjoeberg, A. A. Leyrat, D. M. Pirone, C. S. Chen and S. R. Quake, Anal. Chem., 2007, 79, 8557-8563.
148 J. Kneipp, H. Kneipp and K. Kneipp, Chem. Soc. Rev., 2008, 37, 1052-1060.

149 K. Kneipp, Y. Wang, H. Kneipp, L. T. Perelman, I. Itzkan, R. Dasari and M. S. Feld, Phys. Rev. Lett., 1997, 78, 1667-1670.

150 A. Walter, A. Maerz, W. Schumacher, P. Roesch and J. Popp, Lab Chip, 2011, 11, 1013-1021.

151 R. Wilson, P. Monaghan, S. A. Bowden, J. Parnell and J. M. Cooper, Anal. Chem., 2007, 79, 7036-7041.

152 I. F. Cheng, C.-C. Lin, D.-Y. Lin and H. Chang, Biomicrofluidics, 2010, 4, 034104.

153 Y. S. Huh, A. J. Lowe, A. D. Strickland, C. A. Batt and D. Erickson, J. Am. Chem. Soc., 2009, 131, 2208-2213.

154 H. Park, S. Lee, L. Chen, E. K. Lee, S. Y. Shin, Y. H. Lee, S. W. Son, C. H. Oh, J. M. Song, S. H. Kang and J. Choo, Phys. Chem. Chem. Phys., 2009, 11, 7444-7449.

155 Y. Ueda, S. Z. Wang, N. Dumont, J. Y. Yi, Y. Koh and C. L. Arteaga, J. Biol. Chem., 2004, 279, 24505-24513.

156 D. Choi, T. Kang, H. Cho, Y. Choi and L. P. Lee, Lab Chip, 2009, 9, 239-243.

157 A. W. Martinez, S. T. Phillips, E. Carrilho, S. W. Thomas, III, H. Sindi and G. M. Whitesides, Anal. Chem., 2008, 80, 3699-3707.

158 A. W. Martinez, S. T. Phillips and G. M. Whitesides, Proc. Natl. Acad. Sci. U. S. A., 2008, 105, 19606-19611.

159 Y. Lu, W. Shi, L. Jiang, J. Qin and B. Lin, Electrophoresis, 2009, 30, 1497-1500.

160 J. Yu, L. Ge, J. Huang, S. Wang and S. Ge, Lab Chip, 2011, 11, 1286-1291.

161 J. Yu, S. Wang, L. Ge and S. Ge, Biosens. Bioelectron., 2011, 26, 3284-3289.

162 Z. Nie, C. A. Nijhuis, J. Gong, X. Chen, A. Kumachev, A. W. Martinez, M. Narovlyansky and G. M. Whitesides, Lab Chip, 2010, 10, 477-483.

163 J. L. Delaney, C. F. Hogan, J. Tian and W. Shen, Anal. Chem., 2011, 83, 1300-1306.

164 A. W. Martinez, S. T. Phillips, G. M. Whitesides and E. Carrilho, Anal. Chem., 2010, 82, 3-10.

165 W. W. Yu and I. M. White, Anal. Chem., 2011, 82, 9626-9630.

166 L.-L. Qu, D.-W. Li, J.-Q. Xue, W.-L. Zhai, J. S. Fossey and Y.T. Long, Lab Chip, 2012, 12, 876-881.

167 J. Zhou, K. Ren, Y. Zhao, W. Dai and H. Wu, Anal. Bioanal. Chem., 2011, 402, 1601-1609.

168 Y. S. Huh, A. J. Chung, B. Cordovez and D. Erickson, Lab Chip, 2009, 9, 433-439.

169 M. Becker, C. Budich, V. Deckert and D. Janasek, Analyst, 2009, 134, 38-40.

170 P. A. Walker, M. D. Morris, M. A. Burns and B. N. Johnson, Anal. Chem., 1998, 70, 3766-3769.

171 L. He, M. J. Natan and C. D. Keating, Anal. Chem., 2000, 72, 5348-5355.

172 R. Keir, E. Igata, M. Arundell, W. E. Smith, D. Graham, C. McHugh and J. M. Cooper, Anal. Chem., 2002, 74, 1503-1508.

173 S. Farquharson and P. Maksymiuk, Appl. Spectrosc., 2003, 57, 479-482.

174 R. M. Connatser, L. A. Riddle and M. J. Sepaniak, J. Sep. Sci., 2004, 27, 1545-1550.

175 R. M. Connatser, M. Cochran, R. J. Harrison and M. J. Sepaniak, Electrophoresis, 2008, 29, 1441-1450. 